\title{
A Model Driven Architecture Approach to Generate Multidimensional Schemas of Data Warehouses
}

\author{
https://doi.org/10.3991/ijoe.v15i12.10720
}

\author{
Amine Azzaoui ${ }^{\bowtie}$, , Ouzayr Rabhi, Ayyoub Mani \\ Mohamed First University, Oujda, Morocco
}

a.azzaouidenim.ac.ma

\begin{abstract}
Over the past decade, the concept of data warehousing has been widely accepted. The main reason for building data warehouses is to improve the quality of information in order to achieve specific business objectives such as competitive advantage or improved decision-making. However, there is no formal method for deriving a multidimensional schema from heterogeneous databases that is recognized as a standard by the OMG and the professionals of the field. Which is why, in this paper, we present a model-driven approach (MDA) for the design of data warehouses. To apply the MDA approach to the Data warehouse construction process, we describe a multidimensional meta-model and specify a set of transformations from a UML meta-model which is mapped to a multidimensional meta-model. The transformation rules are programmed by the Query View Transformation (QVT) language. A case study illustrates our approach. It demonstrates how it reinforces the components traceability and reusability and how it globally improves the modeler's efficiency. Furthermore, the use of the UML, as a technique to build data warehouses, is an important facilitator which prepares our further work to automate this approach.
\end{abstract}

Keywords-Data Warehouse, Meta model, Model Driven Architecture, Transformation, UML

\section{Introduction}

The decision-making systems are defined by information systems that can help companies in their decision making process. To support these decisions, it has become necessary to manage and analyze large amounts of data to obtain recent and relevant knowledge. In this sense, a new concept of database was created: Data warehouse. In the last decade, the data warehouse concept has experienced great acceptance. More than improving information quality, data warehouse also enhance decision making by providing a complete analysis of the organization, its business and needs.

Data Warehouse (DW) systems provide a Multidimensional (MD) view of huge amounts of historical data from heterogeneous operational sources, thus supplying useful and sensitive information which allows decision makers to improve organizations' business processes [1]. However, building a DW is still a challenging and complex task 
because it consists of several interrelated components with different functions, different design pitfalls, and different technologies [2].

To overcome this problem, we decided to use the Model Driven Architecture (MDA) approach in building the DW which is developed by Object Management Group (OMG), supports evolving standards in application domains. MDA provides an open, vendor-neutral approach to the challenge of interoperability, building upon and leveraging the value of OMG's established modeling standards: Unified Modeling Language (UML); Meta-Object Facility (MOF) [3]. This framework separates the specification of system functionality in a Platform Independent Model (PIM) from the specification of the implementation of that functionality on a specific technology in a Platform Specific Model (PSM). Furthermore, the system requirements are specified in a Computation Independent Model (CIM). MDA does not only allows the development of these models in a formal and integrated way by using a standard notation, but also the specification of model transformations in order to obtain the final software product [4]. The main benefit of MDA is that less time and effort are needed to develop the whole software system, thus improving productivity. Furthermore, MDA provides support for system evolution, integration, interoperability, portability, adaptability and reusability [5]-[7].

On the other hand, to help with the decision making process, and to measure data warehouse performances financially and non-financially, especially since the benefits of data warehouse are obscured and calculation of the return on investment (ROI) of data warehousing is in most cases infeasible [8], we opted for the use of the Balanced Scorecard (BS) Framework. At present balanced scorecards is being used as an organizational performance measuring tool which measures the company from four different perspectives to transform the strategy in to action in the critical divisions of a company [9].

Considering all this aspects, the present paper describes a model driven approach for the development of DWs based on MDA principles. We focused on the PIM to PIM transformation using an approach by modeling, we will automatically generate DW schema by using several UML profiles. The resulting PIM represent conceptual model of DW component, without any reference to a concrete target platform or a specific technology. We developed as well the transformation rules by using formal QVT transformations. This type of processing is used to enrich, filter or specialize model information without adding any information related to the platform.

The present paper is structured as follows. Section 2 presents the most relevant related work. In section 3 we outline the Background knowledge. Sections 4 introduces our MDA approach for generating the MD modeling of the DW repository. A case study is presented in Section 5. Finally, section 6 concludes the work and offers further perspectives.

\section{Related Work}

In this section, we present a brief overview of some approaches, proposed for the development of DW systems. 
In [8], the authors propose a Fact-Dimension model. It is a graphical conceptual model for DW. They also show how to derive a DWH schema from the data sources described by the entity-relationship diagram.

A multidimensional conceptual Object-Oriented model for Data Warehousing is proposed in [9]. It was developed as an extension of the UML notation to represent the multi-dimensional data structure. The authors also present OLAP (OnLine Analytical Processing) tools, its structures, integrity constraints and query operation but they don't show how to get the presented conceptual model.

In [1], the authors are interested in secure XML data warehouses. They propose an approach for the model driven development of these DW in which the secure conceptual DW data model, the PIM, is transformed into a secure XML DW, as a PSM, by applying a set of transformation rules.

In [4], the authors present an MDA approach for the development of the DW repository, and then, describe how to build the different MDA models for the DW repository by using an extension of UML and the Common Warehouse Meta model (CWM). They modeled the PIM by using their UML profile for the multidimensional modeling of DW. They derive then the PSM taking into account the deployment platform. From this PSM, the necessary SQL code is derived to create data structures for the DW in a relational platform.

In [10], a well-structured approach is proposed to formalize the development of the DW repository. Authors establish a set of multidimensional normal forms in order to obtain a conceptual model of the DW repository. A relational representation from the conceptual model is informally derived, but no formal transformations are defined to obtain the logical model.

\section{Background Knowledge}

\subsection{Model driven engineering}

In late 2000, OMG, a consortium of over 1,000 companies, first reviewed the document entitled "Model Driven Architecture" and decided to form an architecture team to produce a more formal statement of the MDA [3]. This approach focuses on developing the highest level of abstraction models and promotes the transformation approach from one model to another.

MDA addresses the challenges of today's highly networked, constantly changing systems, providing an architecture that assures portability, cross-platform Interoperability, platform independence, domain specificity and productivity [3]. The key to the MDA approach is the importance of models in the software development process. In the MDA, the software development process is driven by the modeling activity of the software system.

In the field of software engineering, the OMG with its MDA approach classifies four types of models that it advocates for the construction of software: Computation Independent Model (CIM), Platform Independent Model (PIM), Platform Specific Model (PSM) and Code; defined as follow: 
- CIM: The goal is to create a requirements model for the future application. Such a model must represent the application in its environment in order to define the services offered by the application and which other entities with which it interacts.

- PIM: This model represents the system-specific business logic or the design model. It represents the functioning of entities and services. It must be durable and lasting over time. It describes the system, but does not show the details of its use on the platform.

- PSM: MDA considers that the code of an application can be easily obtained from these models. The main difference between a code model and an analysis or design model is that the code model is linked to an execution platform.

The reason for the above model organization is to develop models of the systems' business logic independently from the platforms of execution, then to transform these models automatically to models dependent of the platforms. The complexity of the platforms does no longer appear in the business logic models but it' is found in the transformation [11].

The required steps during the model-driven development with the UML approach can basically be divided into the following steps [12], at first building the CIM that acquires user requirements. Then, according to this CIM, a PIM is built. Next is, the transformation of the proposed PIM into one or more PSMs. This type of transition from CIM to PIM and PIM to PSM is called Model To Model (M2M) transformation. The final step is to transform the generated model respecting the PSM into the code of the chosen platform. This transition is called Model to Text (M2T) transformation. Fig. 1 , shows how the transformations are done.

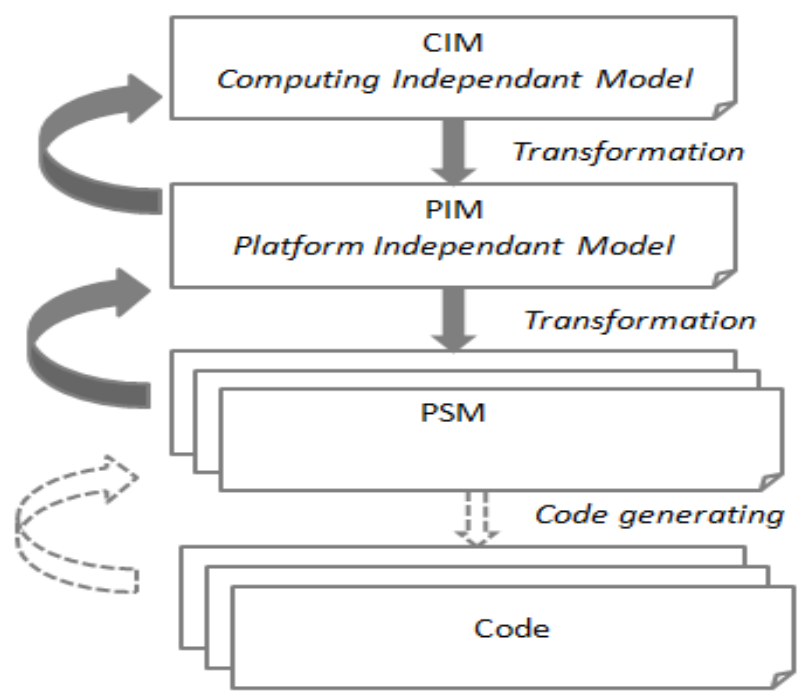

Fig. 1. Model Driven Architecture levels 
The OMG, in the context of MDA, gives the following definition to the transformation of models "the process of converting a model into another model of the same system". In general, it can be said that a transformation definition consists of a collection of transformation rules, which are unambiguous specifications of how a model can be used to create another model. There are three approaches in MDA to perform these transformations:

- Approach by programming: Consists in using object-oriented programming languages. The idea is to program a transformation of models in the same way that any computer application is programmed.

- Approach by template: Consists in defining the canvas of the desired target models by declaring parameters. These parameters will be substituted by the information contained in the source models. The execution of a transformation involves taking a template and replacing its parameters with the values of a source model.

- Approach by modeling: Consists in applying the concepts of the engineering of the models to the transformations of the models themselves. The objective is to model the transformations of models and to make the transformation models perennial and productive and to express their independence vis-à-vis the execution platforms.

Using the modeling approach is designed to have a sustainable and productive models' transformation, independently of any execution platform. This is why the OMG has developed a standard for this transformation language which is the MOF 2.0 QVT [14], standing for Query View Transformation.

\subsection{Data warehouses}

In the early 1990s, as a consequence of a competitive world, organizations realized that data analysis needs to be performed to support their decision making processes. Traditional transactional databases do not satisfy the requirements for data analysis because they are designed to support daily operations and ensure concurrent access. However, they do not include historical data and are not optimized to execute complex queries that involve large volumes of data. Therefore, data warehouses were proposed as a solution to overcome these problems and limitations [15]. A data warehouse provides an infrastructure that allows users to get efficient and accurate responses to complex queries. Different systems and tools can be used to access and analyze data stored in data warehouses.

A data warehouse is a subject-oriented, integrated, non-volatile, and time-variant collection of data in support of management's decisions [16]:

- Subject-oriented: The Data Warehouse is organized around major topics of the company such as: customer, sales, products... This organization necessarily affects the design and implementation of data in the Data Warehouse.

- Integrated: Data is integrated from various operational and external systems.

- Non-volatile: Data is accumulated from operational systems for a long period of time. 
- Time-variant: In a decision-making system it is important to keep the different values of a given data, allowing comparisons and monitoring of the evolution of values over time, the Data Warehouse keeps track of how its data evolved over time.

Fig. 2, describes a typical architecture of a DW system, which consist of several tiers [17]:

- The back-end tier is composed of extraction-transformation-loading (ETL) tools, used to feed data in from operational databases and other data sources, and a data staging area, which is an intermediate database.

- The DW tier is composed of an enterprise data warehouse and/or several data marts, and a metadata repository.

- The OLAP tier is an OLAP server that supports multidimensional data and operations.

- The front-end tier contains client tools such as reporting tools and data-mining tools.

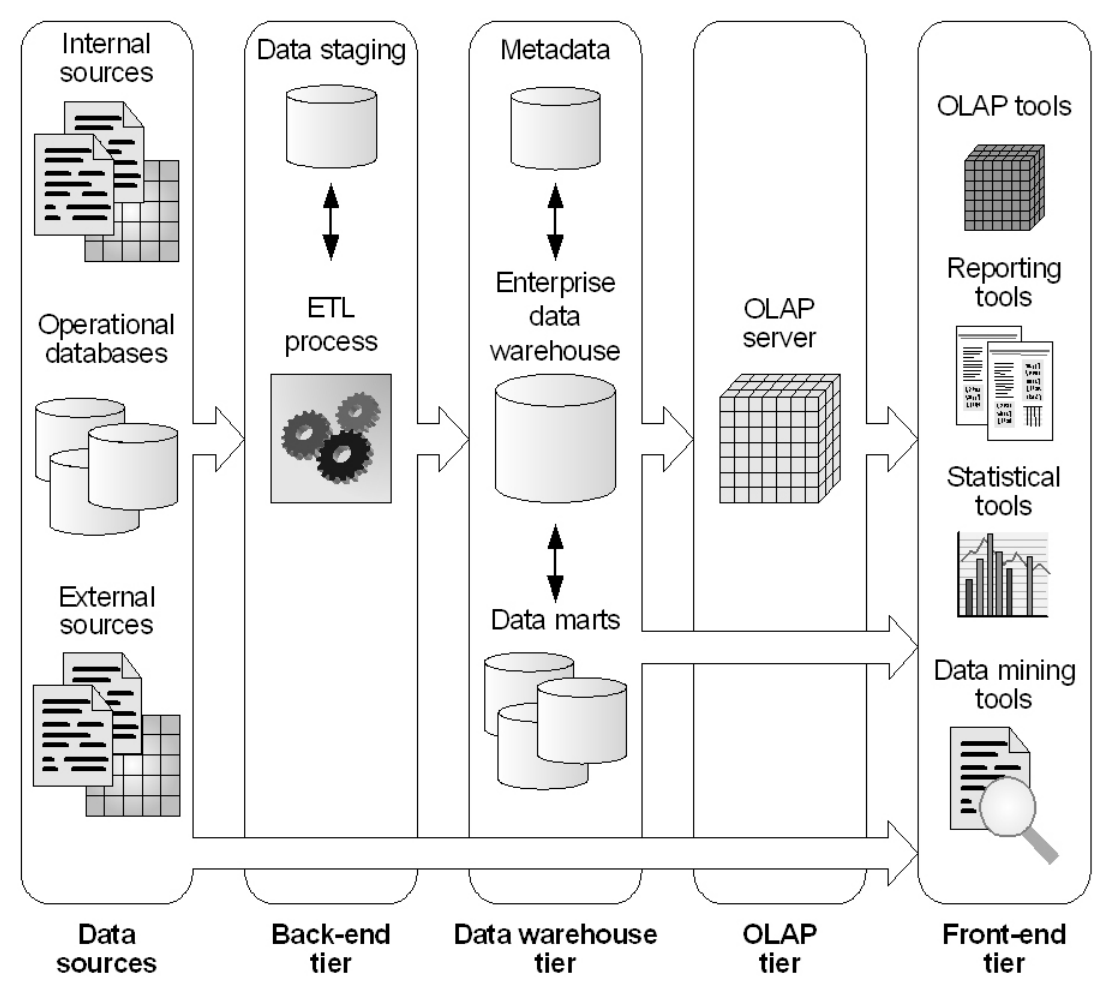

Fig. 2. Typical data warehouse architecture

\subsection{BSC strategy}

The creation of a strategic and balanced dashboard may be based upon various reference models. The most diffused and renowned model is the Balanced Scorecard 
(BSC). The basic concepts of the BSC were elaborated by Kaplan and Norton in [21]. After that, the concepts were developed in numerous publications between 1996 and 2008. Multinational companies with a multi-division organization are the main context of reference of Kaplan and Norton [22].

The balanced scorecard suggests that we view the organization from four perspectives, and to develop metrics, collect data and analyze it relative to each of these perspectives [9]:

The Learning \& Growth Perspective: It includes employee training and corporate cultural attitudes. It is becoming necessary for workers to be in a continuous learning mode. Kaplan and Norton said that learning is more than training, it also includes things like mentors and tutors within the organization. Moreover, technological tools may be included.

The Business Process Perspective: It refers to internal business processes. Metrics based on this perspective allow the managers to get answers to two questions: how well the business is running? And are the products and the services conform to customer requirements?

The Customer Perspective: Recent management philosophy has shown that customer focus and customer satisfaction has taken an increasingly important role in any business. If customers are not satisfied, they will eventually find other suppliers that will meet their needs. Thus, in developing metrics for satisfaction, customers should be rigorously analyzed in order to ensure high performance from this perspective.

The Financial Perspective: Even though managers try to provide timely and accurate funding data, the emphasis on financials leads to an unbalanced situation with regard to other perspectives.

\section{MDA for MD Modeling of DW}

In this paper we proposed a meta model as a PIM to describe DW. Then, we chose to transform a UML model to PIM, with an approach by modeling using QVT. This type of transformation will allow us to automatically generate the multidimensional data warehouse schema from UML schema. We used UML in our approach, because it takes into account the structural and dynamic properties of the information system at a conceptual level. The class diagram of analysis is obtained from the data dictionary and functional dependencies. Obtaining the conceptual class diagram is based on scenarios of different use cases.

\subsection{The modeling process}

PIM Source Meta Model: The UML specification has the Kernel package which provides the core modeling concepts of the UML, our source Meta-model structures a simplified UML model based on a package containing the data types and classes. The classes contain structural features represented by attributes, and behavioral features represented by operations [13]. Fig. 3, presents the source meta model. 


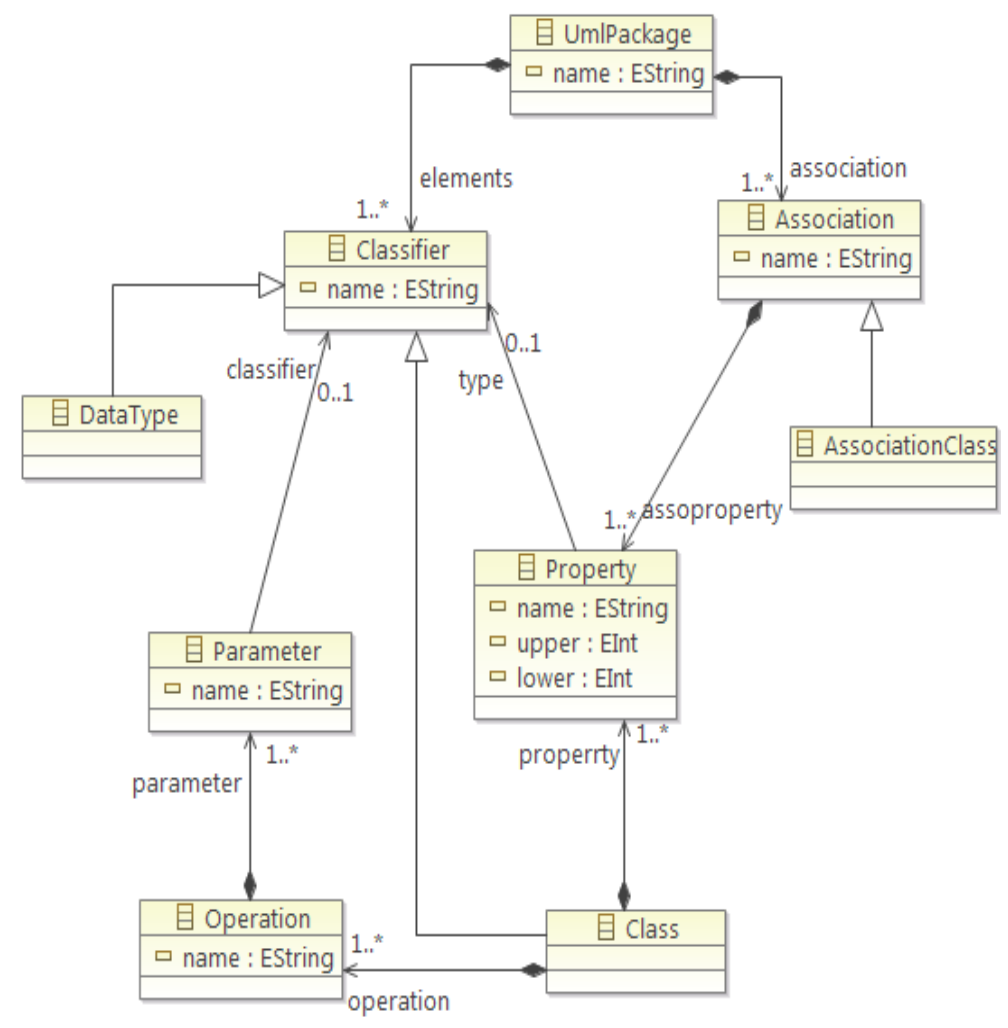

Fig. 3. Simplified UML Meta-Model

- Uml Package: Uml Package is an abstract element of UML used to group together elements that are semantically related. This meta-class is connected to the meta-class Classifier.

- Classifier: Classifier is an abstract meta-class which describes set of instances having common features. This meta-class represents both the concept of class and the concept of data type.

- Class: Class represents the concept of UML class.

- Data Type: Data Type represents the data type of UML.

- Operation: Operation expresses the concept of operations of a UML class.

- Parameter: Parameter describes the order, type and direction of arguments that can be given when the operation is invoked. It explains the link between Parameter metaclass and Classifier meta-class.

- Property: Property expresses the concept of Property of a UML class. These properties are represented by the multiplicities and meta-attributes upper and lower. A UML class is composed of properties, which explains the link between the metaclass Property and meta-class Class. These properties can be primitive type or class type. This explains the link between the meta-class and the Classifier meta-class Property. 
PIM Target Meta Model: Based on the approach described in [18], and inspired by this UML profile for the data warehouse, the target meta-model that we have proposed for the transformation, is a simplified meta-model containing Facts and Dimensions. A Fact contains attributes related to dimensions. This meta model is an extension of the work done by the authors in [19]. Fig. 4, shows the Target Meta Model:

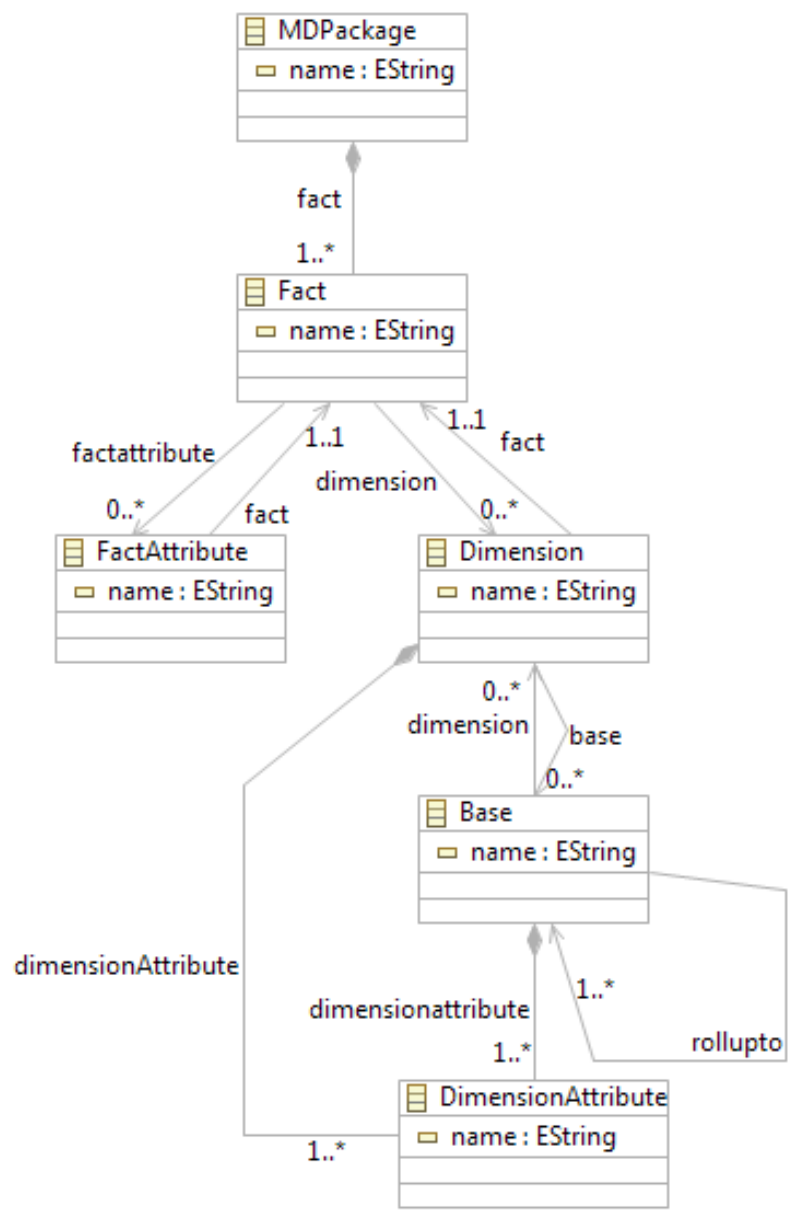

Fig. 4. Simplified Meta-Model of multidimensional schema

- MD Package: MD Package expresses the concept of the package; it contains Fact classes.

- Fact: Fact expresses the concept of Fact in a multidimensional schema. Each fact is contained in MD Package, which explains the link between MD Package and Fact.

- Fact Attribute: Fact Attribute expresses the concept of attributes for the element Fact and Fact contains Fact Attribute. 
- Dimension: Dimension expresses the concept of dimension in a multidimensional schema.

- Dimension Attribute: Dimension Attribute expresses the concept of dimensions attributes. A dimension contains attributes, which explains the link between a Dimension Attribute and Dimension.

- Base: Base is the basic concept in the multidimensional schema. A base represents the hierarchical level of classification. An association (represented by the ROLLSUPTO stereotype) between the base class specifies the relationship between two hierarchical levels of classification.

\subsection{The transformation process}

Once the meta models developed, the next step is to specify the correspondences between the two meta models and automatically generate DW model from the UML model by using transformation rules written in QVT Operational Mappings. In this section we'll explain some of the programmed transformation rules.

A QVT transformation is in the form of a function with two parameters, the first corresponds to the source model, the second corresponds to the target model. The main function of our transformations is as follow:

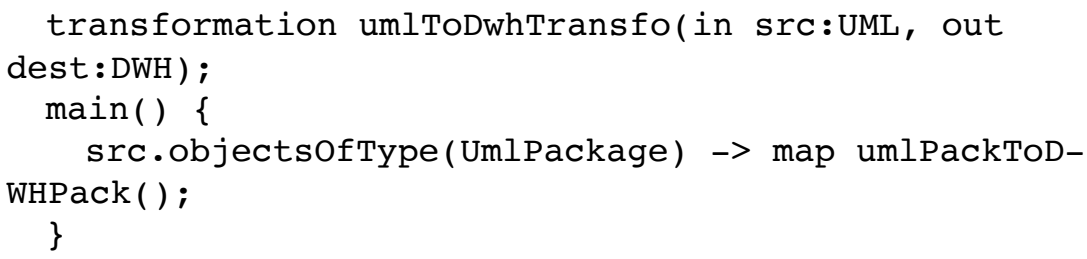

We consider that a simplified UML model consists of a package called UmlPackage. This package will be transformed into a multidimensional package called MDPackage, using the transformation rule defined as follows:

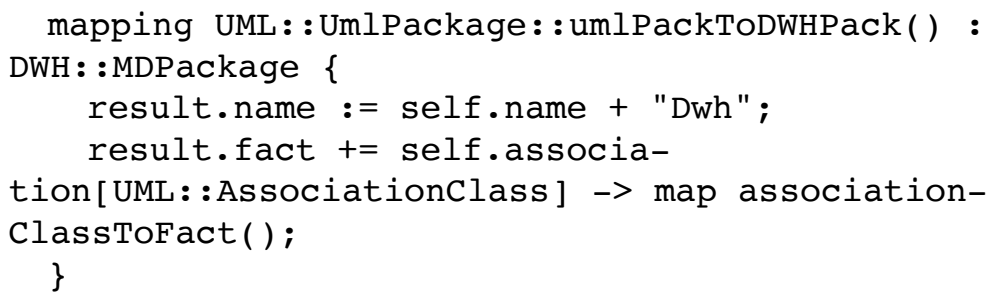

The following code shows the transformation rule of a class association from a UML class diagram to a fact. The fact will carry the name of the association class, its attributes will be the properties of the association class:

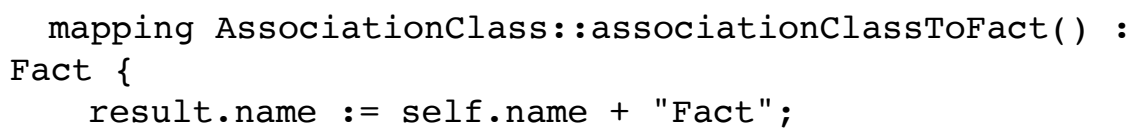




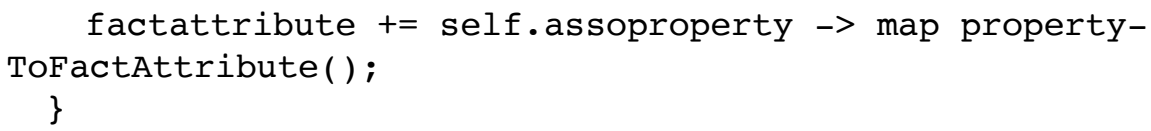

\section{$5 \quad$ Case Study}

To validate the proposed approach, we took the demands concerning construction projects at the urban community of Oujda, Morocco as a case study. To that end, we instantiated the meta model defined earlier. Fig. 5 below presents a the input model:

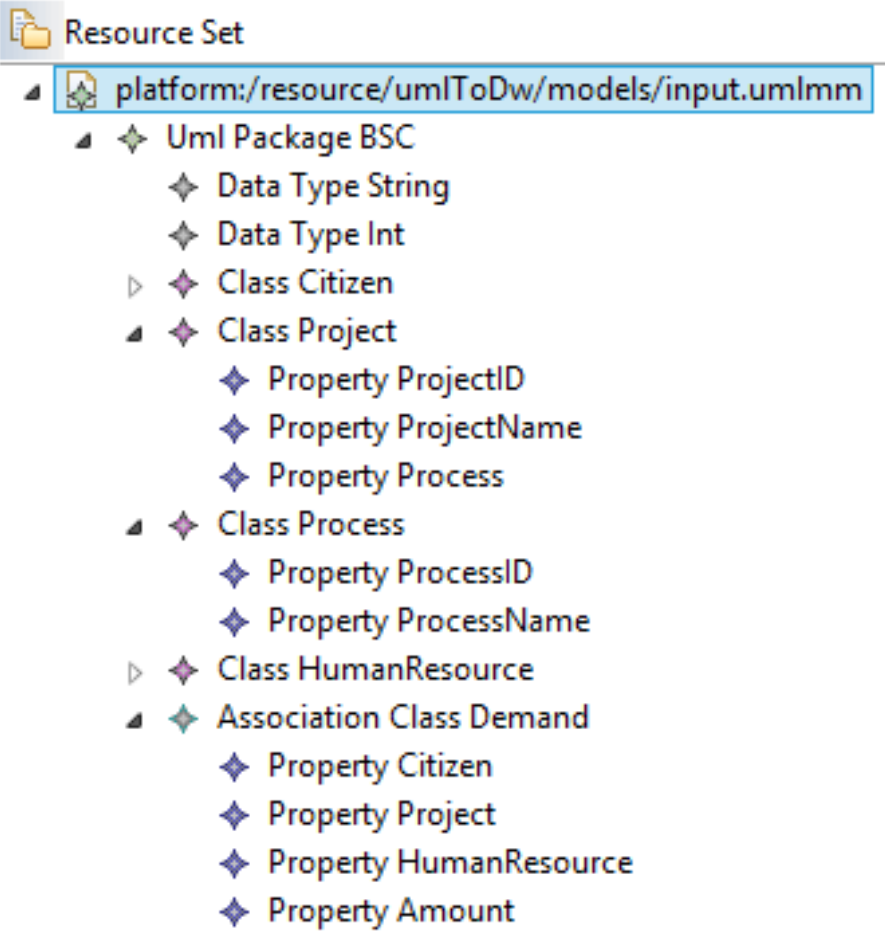

Fig. 5. The input model

The input model includes the four perspectives of the Balanced Score Card strategy:

- Learning \& growth perspective: Human Resource Class.

- Business process perspective: Process Class.

- Customer perspective: Citizen Class.

- Financial perspective: Amount Property. 


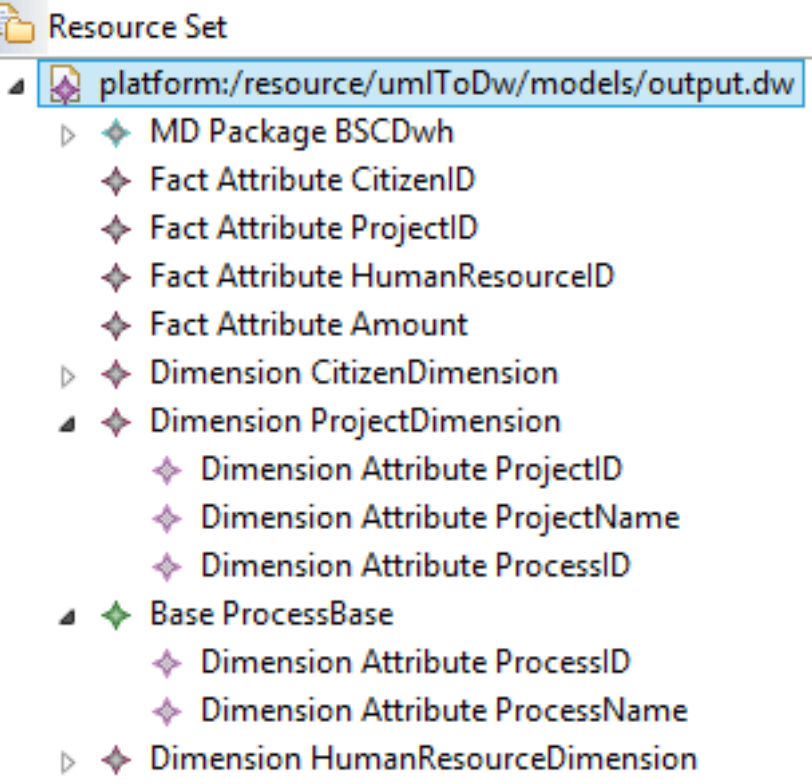

Fig. 6. The tranformation result

\section{Conclusion}

In this paper, we have introduced our MDA approach for the development of DWs. For constructing the system, we applied transformations from a UML PIM in order to automatically obtain an analogous DW PIM. Thanks to the use of MDA and QVT, we can generate from a simplified UML model instance, a simplified Multidimensional model in just two steps: first is the development of the source and target PIMs; and second is the development of the corresponding QVT transformations.

The primary benefit of our approach is that the complex task of designing the whole DW is done in a systematic, well-structured, and standard way by using an MDA approach.

Our future intentions include implementing our MDA approach for the development of DWs into specific platforms by creating the corresponding PSMs. And transforming each generated PSM into code.

\section{$7 \quad$ References}

[1] B. Vela, C. Blanco, E.F. Medina, E. Marcos, A Practical Application of our MDD Approach for Modeling Secure XML Data Warehouses, (2012) Decision Support Systems (DSS), 54 (4), pp. 899-925. https://doi.org/10.1016/j.dss.2011.11.008

[2] R. Kimball, M. Ross, The Data Warehouse Toolkit, $3^{\text {rd }}$ Edition (John Wiley \& Sons, Inc. 2013). 
[3] Object Management Group (OMG), MDA Guide 2.0. http://www.omg.org/cgi-bin/doc? ormsc/14-06-01

[4] J.N. Mazón, J. Trujillo, An MDA Approach for the Development of Data Warehouses, (2008) Decision Support Systems (DSS), 45 (1), pp. 41-58. https://doi.org/10.1016/j.dss. 2006.12.003

[5] D.S. Frankel, Model Driven Architecture: Applying MDA to Enterprise Computing (Wiley, 2003).

[6] A.G. Kleppe, J. Warmer, W. Bast, MDA Explained: The Model Driven Architecture: Practice and Promise (Addison-Wesley, 2003).

[7] S. Mellor, K. Scott, A. Uhl, D. Weise, MDA Distilled: Principles of Model-driven Architecture (Addison-Wesley, 2004).

[8] H.J. Watson, B.J. Haley, Managerial Considerations with Data Warehousing, (1998) Communications of the ACM (CACM), 41 (9), pp. 37-37. http://dx.doi.org/10.1145/28507 $\underline{0.285077}$

[9] Balanced Scorecard Institute. http://balancedscorecard.org/Resources/About-the-Balanced$\underline{\text { Scorecard }}$

[10] M. Golfarelli, D. Maio, S. Rizzi, The Dimensional Fact Model: a Conceptual Model for Data Warehouses, (1998) International Journal of Cooperative Information Systems (IJCIS), 7 (2\&3), pp. 215-247. https://doi.org/10.1142/s0218843098000118

[11] A. Abelló, J. Samos, F. Saltor, YAM2: a Multidimensional Conceptual Model Extending UML, (2006) Information Systems (IS), 31 (6), pp. 541-567. https://doi.org/10.1016/ j.is.2004.12.002

[12] J. Lechtenbörger, G. Vossen, Multidimensional Normal Forms for Data Warehouse Design, (2003) Information Systems (IS), 25 (5), pp. 415-434. https://doi.org/10.1016/s03064379(02)00024-8

[13] S. Abdoli, S. Kara, Designing Warehouse Logical Architecture by Applying Object Oriented Model Based System Engineering, (2016) Procedia CIRP, 50, pp. 713-718. https://doi.org/10.1016/j.procir.2016.04.108

[14] X. Blanc, MDA en Action: Ingénierie Logicielle Guidée par les Modèles (Eyrolles, 2005).

[15] S. Roubi, M. Erramdani, S. Mbarki, Generating Graphical User Interfaces Based on Model Driven Engineering, (2015) International Review on Computers and Software (IRECOS), 10 (5), pp. 520-528. https://doi.org/10.15866/irecos.v10i5.6303

[16] S. Mbarki, M. Erramdani, Model-Driven Transformations: From Analysis to MVC 2 Web Model, (2009) International Review on Computers and Software (IRECOS), 4 (5), pp. 612 620. https://doi.org/10.15866/irecos.v9i9.3361

[17] Object Management Group (OMG), MOF 2.0 QVT. http://www.omg.org/spec/MOF/2.0/ PDF

[18] A. Vaisman, E. Zimányi, Data Warehouse Systems: Design and Implementation (Springer Berlin Heidelberg, 2014). http://dx.doi.org/10.1007/978-3-642-54655-6

[19] W. H. Inmon, Building the Data Warehouse (John Wiley \& Sons, 2002).

[20] E. Malinowski, E. Zimányi, Advanced Data Warehouse Design: From Conventional to Spatial and Temporal Applications (Springer-Verlag Berlin Heidelberg, 2008). https://dx.doi.org/10.1007/978-3-540-74405-4

[21] R. S. Kaplan, D. P. Norton, The Balanced Scorecard - Measures that Drive Performance, (1992) Harvard Business Review (HBR), 69 (1), pp. 71-79.

[22] S. Biazzo, P. Garengo, Performance Measurement with the Balanced Scorecard: A Practical Approach to Implementation within SMEs (Springer-Verlag Berlin Heidelberg, 2012). https://dx.doi.org/10.1007/978-3-642-24761-3 
[23] S.L. Mora, J. Trujillo, I.Y. Song, A UML Profile for Multidimensional Modeling in Data Warehouses, (2006) Data \& Knowledge Engineering, 59 (3), pp. 725-769. https://doi.org/ 10.1016/j.datak.2005.11.004

[24] I.Arrassen, A.Meziane, R.Sbai, M.Erramdani, QVT Transformation by Modelling - From UML Model to MD Model, (2011) International Journal of Advanced Computer Science and Applications (IJACSA), 2 (5), pp. 7-14. https://doi.org/10.14569/ijacsa.2011.020502

\section{Authors}

Amine Azzaoui is a PhD student from MATSI Laboratory, EST, Mohamed First University, Oujda, BP 473, Morocco. He is graduated with a computer engineering degree from ENSMR (Rabat Superior National School of Mines) in 2016. His research interests include Business Process Management (BPM) and MDA models for the endto-end design of organizations strategic and operational dashboards. E-mail: a.azzaoui@enim.ac.ma

Ouzayr Rabhi is a Ph.D. student from MATSI Laboratory, EST, Mohamed First University, Oujda, BP 473, Morocco. He holds a Master's degree in Computer Engineering from Oujda Faculty of Science. His research focuses on the management of public organization and MDA models for the end-to-end design of strategic organizations and dashboards of public organization. E-mail: o.rabhi@ump.ac.ma

Ayyoub Mani is a computer engineer. Graduated on 2017 from the National School of Applied Sciences, Oujda, Morocco. He is now preparing a PhD in Model driven architecture. He is with MATSI Laboratory, EST, Mohamed First University, Oujda, BP 473, Morocco. E-mail: maniayyoub@gmail.com

Article submitted 2019-04-22. Resubmitted 2019-06-15. Final acceptance 2019-06-23. Final version published as submitted by the authors. 